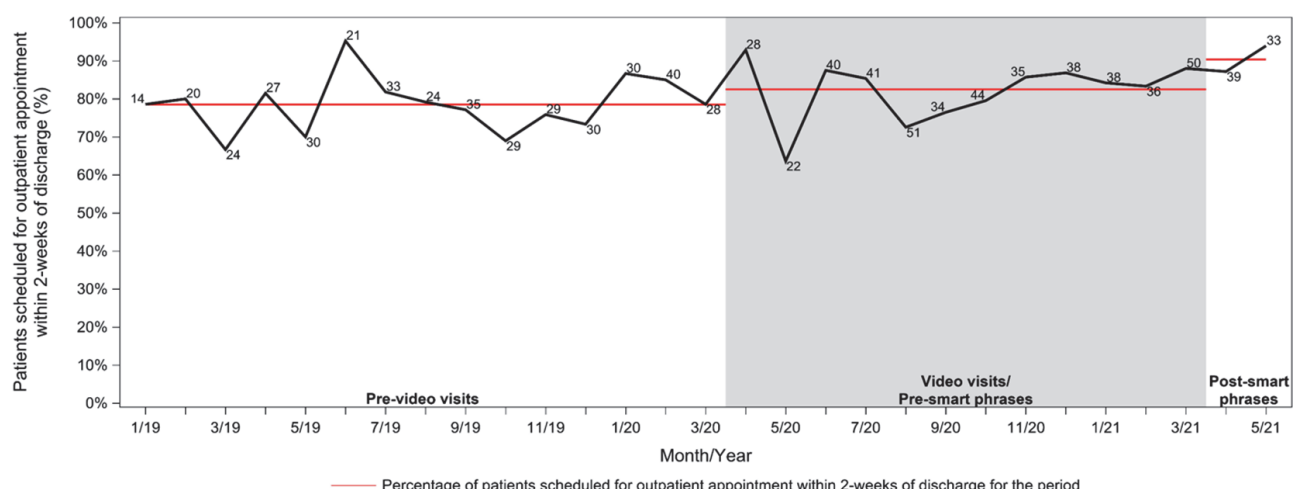

Abstract 17 Figure 4 Impact of video visits and SmartPhrases on percent of patients scheduled for post-discharge appointment within 2 weeks

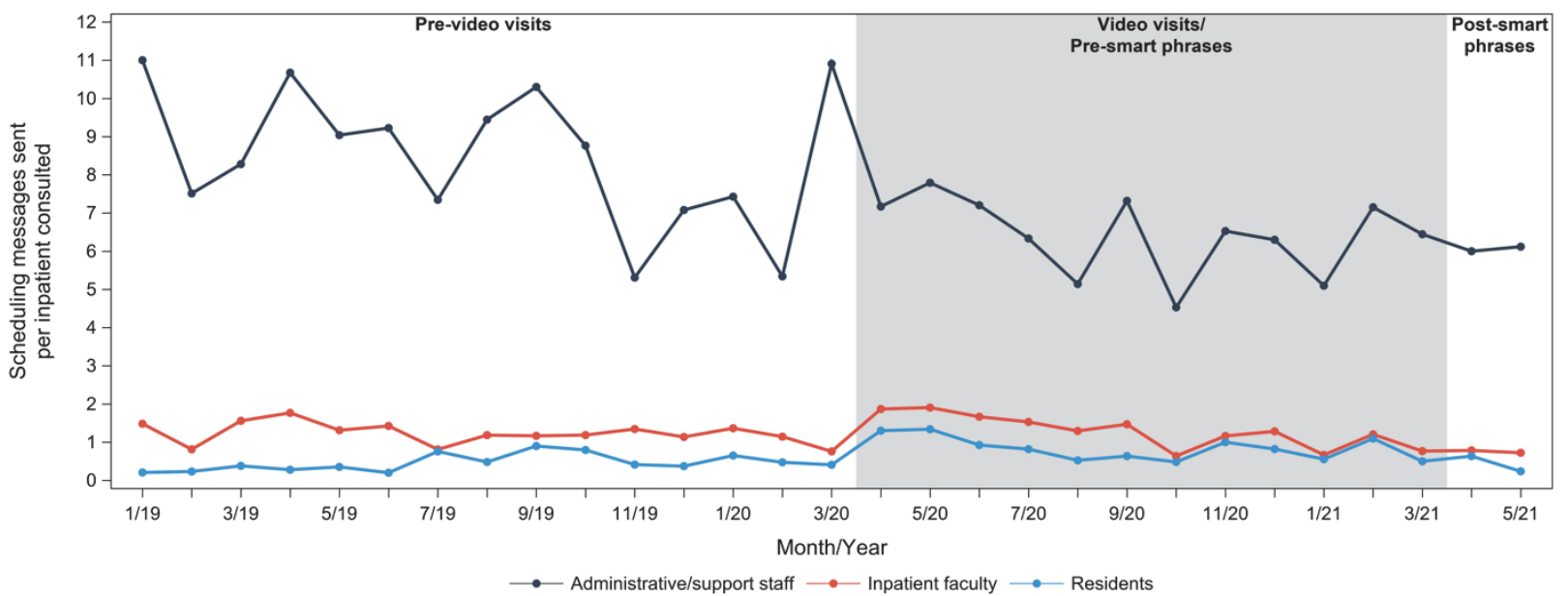

Abstract 17 Figure 5 Impact of video visits and SmartPhrases on number of scheduling messages sent by three stakeholders (administrative/ support staff, inpatient faculty and residents)

Conclusions Early data suggest decreased time to post-discharge scheduling and increased percentage scheduled within two weeks post-VV implementation, and further improvement post-SmartPhrases. Staff messages did not decrease significantly; we plan to track messaging rates through August to fully assess SmartPhrase impact.

\section{THE INTERSECTION OF QUALITY IMPROVEMENT AND IMPLEMENTATION SCIENCE: A CASE STUDY}

${ }^{1}$ Mary A Dolansky, ${ }^{2}$ Anne Pohnert. ${ }^{1}$ Case Western Reserve University; ${ }^{2}$ CVS/MinuteClinic 10.1136/bmjoq-2021-IHI.18
Background Implementing evidence-based practice requires both quality improvement and implementation science to promote adoption and sustainability.

Objectives To present a case study of the implementation of The John A. Hartford Foundation and Institute for Healthcare Improvement supported evidence-based Age-Friendly Health Systems (AFHS) 4Ms Framework: What Matters, Medications, Mentation, and Mobility into the 1,100 MinuteClinics located in 33 states and District of Columbia. Quality improvement methods provided the framework for the initiative that was complimented with research to examine strategies that showed promise for sustainability.

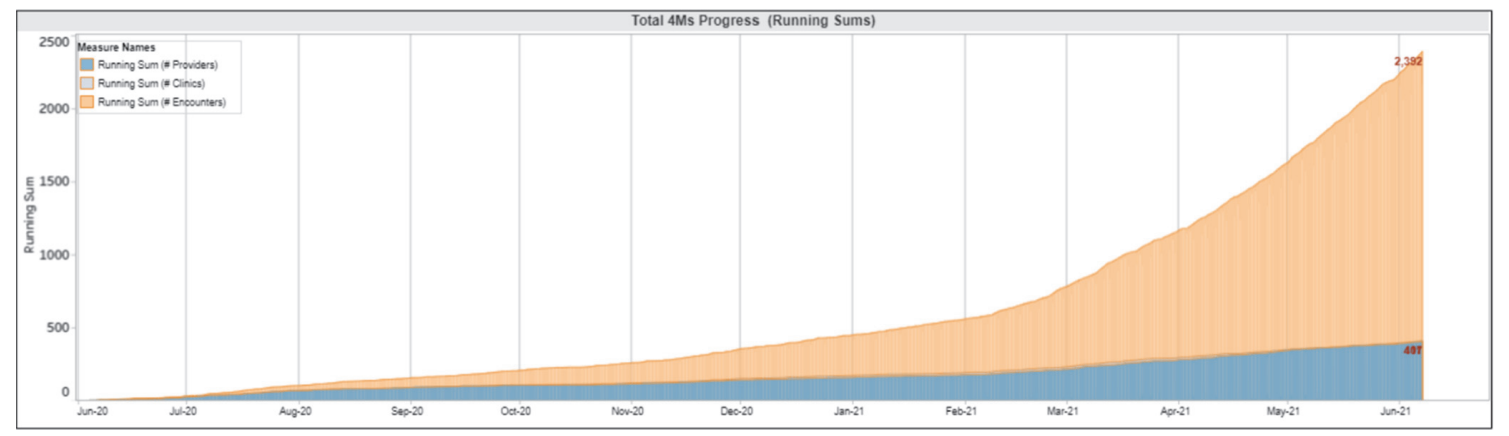

Abstract 18 Figure 1 Running sum of the age-friendly health systems 4Ms assessments and act-ons delivered as a set 
Methods The project was directed by the MinuteClinic Quality Department. A one-year planning period to develop a processflow map, educational materials, practice-based tools, and electronic health record (EPIC) alignment was followed by an 11month implementation phase starting June 1, 2020, in spite of the COVID-19 Pandemic. Due to opportunities for improved uptake, implementation science methods were used synchronously to improve uptake.

Results To date, the 1-year QI project resulted in the adoption of the $4 \mathrm{Ms}$ by 1145 providers (37\%) (figure 1). Continual monitoring of the adoption of the $4 \mathrm{Ms}$ Framework, qualitative methods to understand barriers and facilitators, and testing of

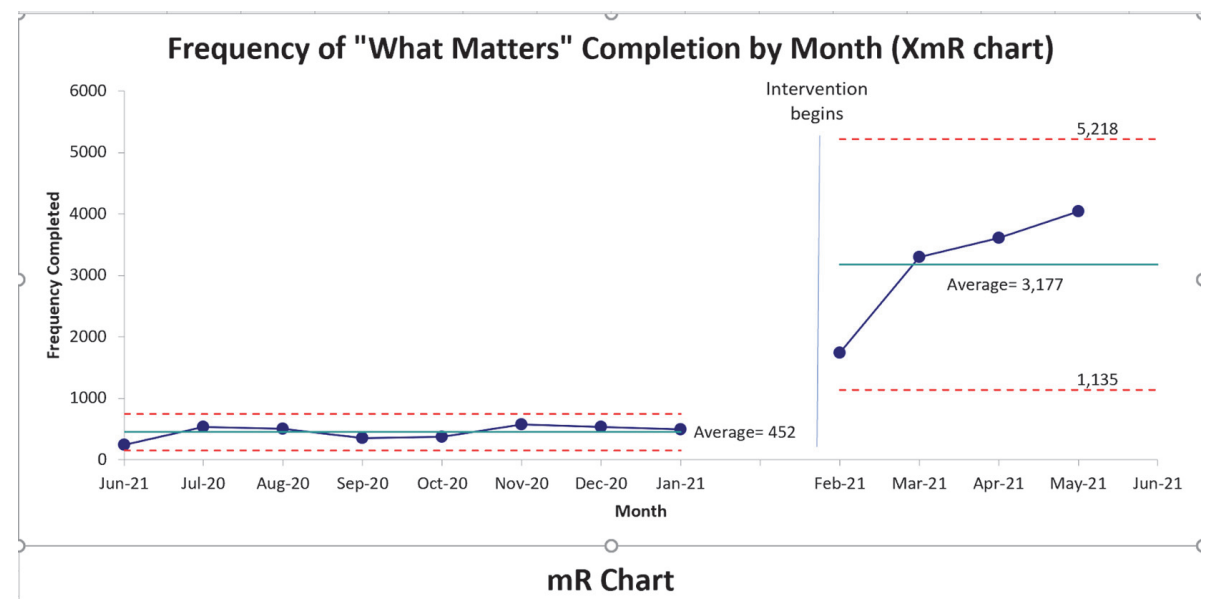

Abstract 18 Figure 2 Statistical process control chart (XMr) of the adoption of what matters

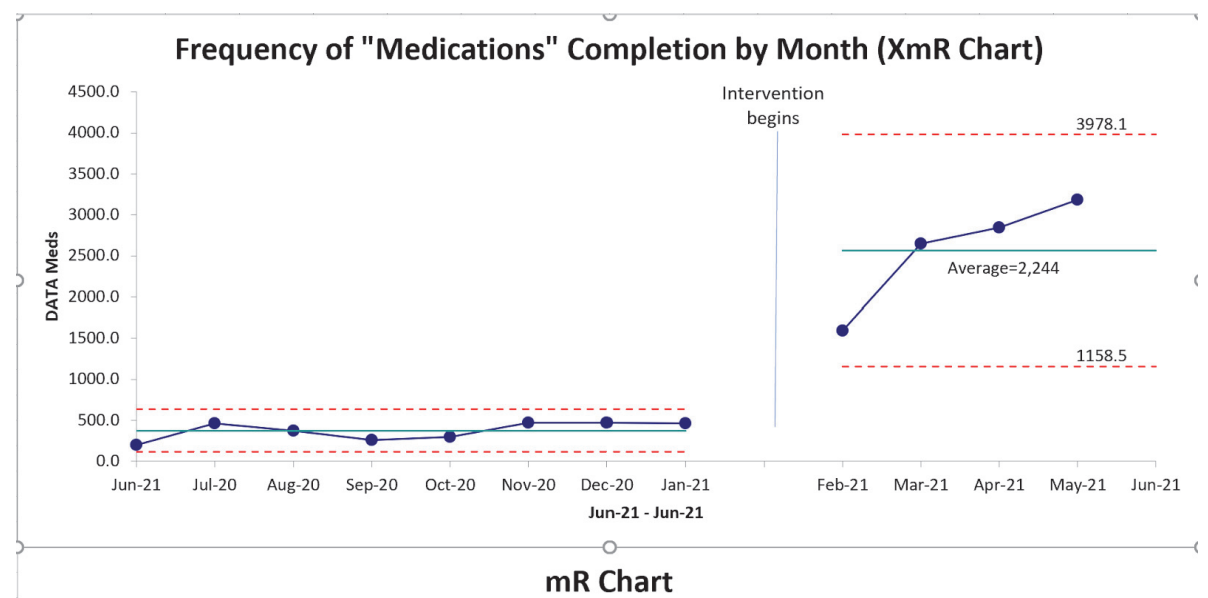

Abstract 18 Figure 3 Statistical process control chart (XMr) of the adoption of medication

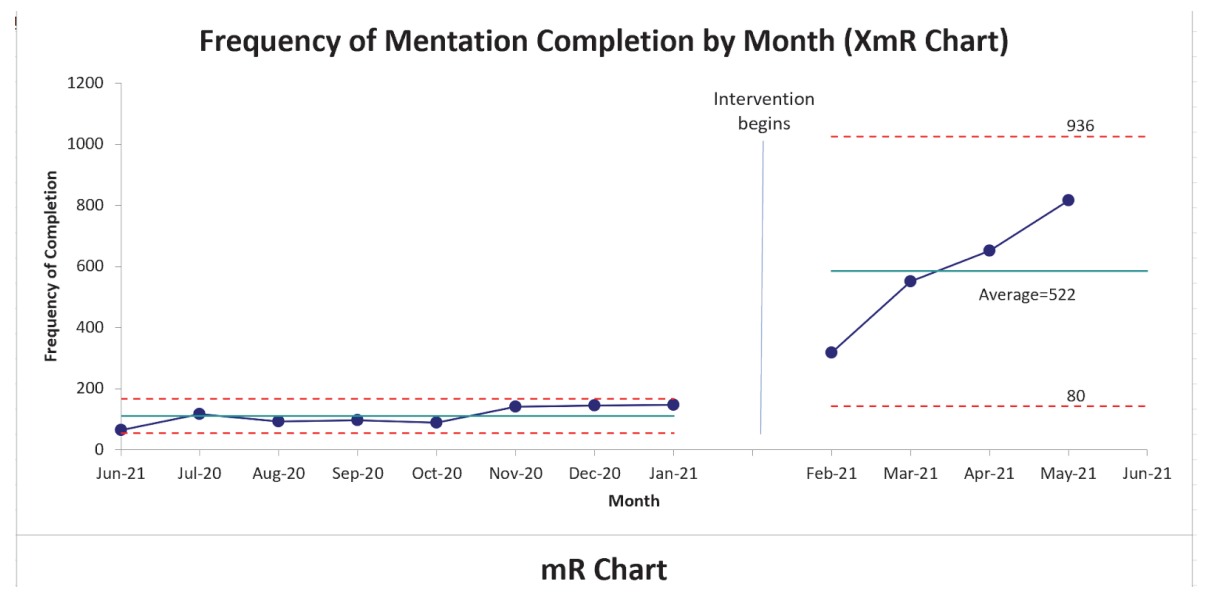

Abstract 18 Figure 4 Statistical process control chart (XMr) of the adoption of mentation 


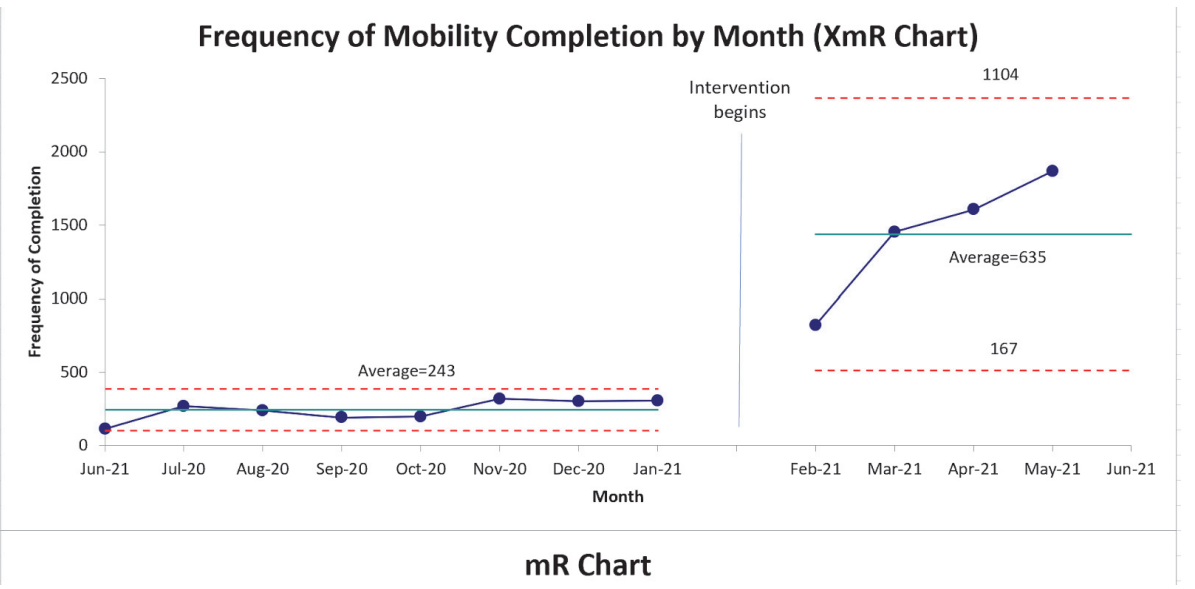

Abstract 18 Figure 5 Statistical process control chart (XMr) of the adoption of mobility

implementation strategies occurred. The project evolved from a local QI project to the study of implementation strategies to understand the mechanism of change strategies. Exploratory analyses using a t-test to examine significant differences in each of the $4 \mathrm{Ms}$ in baseline vs. intervention, significant improvements were noted for all $4 \mathrm{Ms}$ What Matters (figure 2), Medications (figure 3), Mentation (figure 4), and Mobility (figure 5), $\mathrm{p}<0.05$ when comparing the intervention to baseline time periods. Continued efforts to implement booster strategies are underway to ensure that Age-Friendly care is delivered reliably to older adults.

Conclusions Implementation and improvement methods are needed to not only improve the adoption but also to understand implementation strategies to improve uptake and sustainment of the evidence-based Age-Friendly 4Ms Framework.

\section{Featured Poster (FP) abstracts}

\section{IMPROVING UTILIZATION OF PERITONEAL DIALYSIS AMONGST UNDOCUMENTED IMMIGRANTS AT PARKLAND HOSPITAL}

Maria llyas, Kylan Larsen, Joseph Berger. University of Texas Southwestern Medical Center

10.1136/bmjoq-2021-|HI.19

Background Patients with ESRD comprise less than 1\% of the Medicare population, however they are responsible for $7 \%$ of all Medicare spending. Hemodialysis (HD) and peritoneal dialysis (PD) have equivalent clinical outcomes; however, $\mathrm{PD}$ is more cost-effective. Undocumented immigrants, when

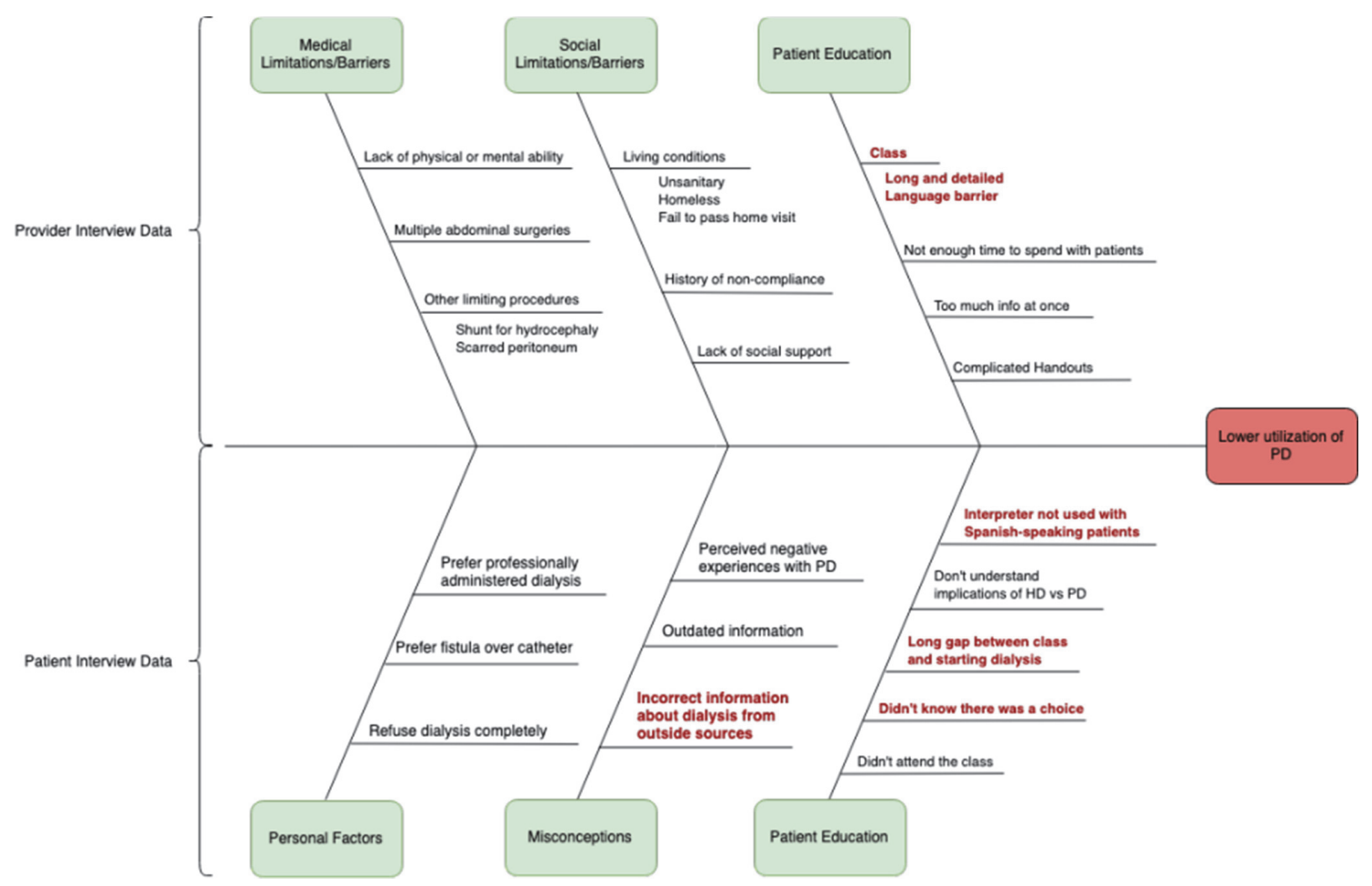

Abstract 19 Figure 1 Fishbone diagram summarizing results from 17 patient interviews and 9 provider interviews (2 nephrologists, 3 NPs, 2 social workers, 2 nurse navigators). Interviews were conducted to understand patient perceptions of PD as a modality and characterize barriers to PD. The top failure modes are highlighted in red. These were utilized for a failure modes and effects analysis (FMEA) which was used to identify top failure causes 